\title{
Entrelacs
}

ENTRELACS Cinéma et audiovisuel

13 | 2017

Espace, perspective et fragmentation

\section{Représentation dissensuelle en architecture}

\section{Daniel ESTEVEZ}

\section{OpenEdition \\ Journals}

Édition électronique

URL : http://journals.openedition.org/entrelacs/2016

DOI : 10.4000/entrelacs.2016

ISSN : 2261-5482

Éditeur

Éditions Téraèdre

Référence électronique

Daniel ESTEVEZ, «Représentation dissensuelle en architecture », Entrelacs [En ligne], 13 | 2017, mis en ligne le 28 février 2017, consulté le 01 mai 2019. URL : http://journals.openedition.org/entrelacs/2016 ; DOI : 10.4000/entrelacs.2016

Ce document a été généré automatiquement le 1 mai 2019.

Tous droits réservés 


\title{
Représentation dissensuelle en architecture
}

\author{
Daniel ESTEVEZ
}

\section{Introduction}

1 Le dessin d'architecture a sédimenté au cours de son évolution différentes capacités à servir le travail de l'architecte qui ont finalement constitué le support traditionnel de son savoir-faire. En analysant certains modes d'adaptabilité d'un système d'information traditionnel de figuration pris en exemple -le dessin géométral- nous tenterons ici de développer les conséquences qu'il est possible d'en tirer concernant l'évolution des pratiques de conception reliées à la figuration architecturale.

2 En particulier, le renouvellement des méthodes contemporaines peut prendre comme point de départ l'usage et l'adaptation dans les systèmes d'information actuels, de certaines procédures figurales traditionnelles qui forment la culture graphique et méthodologique des architectes. Or nous voudrions tenter de souligner que le principe de fragmentation est inhérent à ces procédures traditionnelles de figuration.

Mais n'y a-t-il pas, entre pratiques actuelles et logique traditionnelle de l'usage, des conflits possibles? Plusieurs travaux de recherche en ergonomie ont montré par exemple qu'il n'était pas toujours possible -dans le domaine des situations d'activités instrumentées- de réduire l'écart entre "les schèmes et représentations initiales, spontanées, des sujets et les schèmes et représentations nécessaires pour utiliser l'instrument $»^{1}$. Une voie de réponse à ces problèmes, que nous proposons dans le texte suivant, consiste tout d'abord à identifier les propriétés opératoires et heuristiques des outils traditionnels intégrants à différents niveaux le principe de fragmentation. À partir de là, nous proposons de considérer la notions de dissensus représentationnel, empruntée à Jacques Rancière, comme l'une des orientations d'évolution positive de nos systèmes de représentation actuels pour la conception en architecture. 


\section{La signification des contraintes méthodologiques d'un système d'information traditionnel}

4 Le dessin géométral en plan, coupe et élévation peut véritablement revêtir un caractère de paradigme parmi les systèmes traditionnels de figuration architecturale. En tant que procédé géométrique de projection parallèle, ce mode de représentation relève initialement des catégories du graphisme technique et du dessin mesurable. Sous cet aspect, les géométraux présentent, affirme Yves Deforges, «la propriété de conserver homothétiquement les angles, les formes, les positions et les dimensions relatives de l'original.»' Mais au delà de cette appartenance initiale, le géométral a très souvent été présenté comme un outil fondamental de conception architecturale: «Plans, coupes, élévations sont donc issus non pas d'une théorie géométrique mais de besoins spécifiques à l'architecture. Il s'ensuit que nous sommes en présence d'une double dépendance de la représentation architecturale qui en fait à la fois une technique de conception et une technique de représentation de l'objet à concevoir.»³.

5 Or ce mode si particulier de figuration défini par le système géométral ne paraît pourtant pas approprié à la recherche de cohérence et de coordination que constitue la conception ou même l'analyse du projet. Le dessin géométral, en effet, est caractérisé non seulement par sa bidimensionnalité mais aussi par sa fragmentation (décomposition en plan/coupe/ élévation). Deux propriétés contraignantes qui vont à l'encontre de l'objectif que se fixe chaque architecte lors de l'élaboration de son projet: en contrôler et en maîtriser l'évolution dans sa globalité et sa complexité (volumétrique, compositionnelle etc.). Comment interpréter ce « paradoxe du géométral»?

6 Nous pouvons introduire les termes de cette contradiction à partir d'une première observation générale sur les pratiques de conception induites par les techniques actuelles d'assistance informatique à la conception.

7 Les concepteurs d'outils de CAO pour l'architecture postulent généralement, dans leurs procédés de représentation, la prédominance de la notion de synthèse sur celle de fragmentation et ainsi s'orientent prioritairement vers la tridimensionnalité (3D) au détriment de la bidimensionnalité (2D). La seconde ne représentant qu'une occurrence ou, plus exactement, un sous-ensemble de la première: «Les objets manipulés par les architectes sont essentiellement tridimensionnels», affirmait le chercheur Paul Quintrand, «Bien que la pratique figurative en conception ait pour support le plan, la validation de cette conception dans l'espace est indispensable ainsi que la possibilité de passer instantanément du plan à la coupe ou à la façade et inversement. Les modèles informatiques mis en oeuvre doivent permettre de traiter le projet de la même façon que l'architecte avec sa planche à dessin par aller retour continuel entre le plan et l'élévation, or seul le modèle tridimensionnel peut permettre cela» $»^{4}$. Cette hypothèse, cependant, n'est pas totalement vérifiée par l'examen attentif des dessins produits par les architectes lors de la conception ou de l'analyse d'un projet. Ces derniers ne semblent pas, en effet, préoccupés en premier lieu par une maîtrise volumétrique constante ou totale des objets qu'ils conçoivent. Si bien qu'un projet abouti, aussi paradoxal que cela puisse paraitre, n'est pas nécessairement à leurs yeux un projet dont l'ensemble des caractéristiques tridimensionnelles sont fixées avec exactitude ${ }^{5}$. L'idée d'une supériorité, pour la maitrise du projet, de la représentation tridimensionnelle sur la représentation bidimensionnelle, n'est en fait que l'application au domaine de la figuration architecturale d'un préjugé du 
sens commun (la facilité du premier venu à voir un volume dans un dessin). Nous voudrions préciser à présent pourquoi, pour l'architecte en situation de conception, cet impérialisme du 3D n'est pas le meilleur gage d'efficience de son travail.

\subsection{La contrainte bidimensionnelle : séparation de l'horizontal et du vertical.}

8 Jean Castex avait déjà analysé, dans son travail sur l'œuvre de FL. Wright ${ }^{6}$, comment la méthode de conception de cet architecte est dépendante des systèmes de représentation qu'il utilise «[...] il dessine en géométral, pas en perspective. La perspective vient après, jamais avant; le croquis perspectif est exclu». Il relève ainsi cette réflexion de l'architecte: «Personne n'a jamais élevé un bâtiment digne d'être de l'architecture en commençant par le façonner à son goût sur un croquis perspectif, pour ensuite forcer le plan à s'y plier. De telles méthodes produisent tout juste des décors. Une perspective peut être une preuve, pas l'aliment du travail». Wright conclut dans ces termes : «[...]J'ai tenté d'établir une relation harmonieuse entre mes plans et mes élévations, en considérant les premiers comme la solution et les secondes comme l'expression.».7

9 Cette distinction des rôles particuliers que remplissent chacun des éléments du géométral dans l'appréhension du projet a été observée par Rudolf Arnheim dans une étude sur les problèmes de perception des formes architecturales ${ }^{8}$. Cet auteur y affirme l'existence d'une différence fondamentale opposant le monde de l'action à celui de la vision; « La dimension principale de l'action est le plan horizontal et tout ce qui touche à l'action est principalement révélé par le plan (du géométral). Le domaine de la vision par contre est le plan vertical.»'. On remarque en effet ceci : c'est le plus souvent par sa représentation en plan que "d'un seul coup d'œil nous saisissons l'essence d'un édifice, sa manière d'assumer sa fonction.» Autrement dit, «[...] Étant donné que le plan horizontal est le domaine de l'action; le plan d'un bâtiment nous montre la manière dont ce dernier fonctionne comme objet et organisateur de l'activité de l'homme.[...] Dès lors, le plan révèle la dimension essentielle d'un bâtiment dont l'extension principale symbolise le comportement humain. $\aleph^{10}$.

Le principe de séparation entre action et vision comme composantes fondamentales de la fonction figurative architecturale a été largement souligné et commenté, Walter Benjamin va jusqu'à le généraliser au domaine, pris dans son ensemble, du dessin et de la peinture: "La projection verticale de l'espace fait seulement appel à la puissance figurative du contemplateur; sa projection horizontale aux forces sensori-motrices. Le dessin reproduit le monde de sorte que l'homme puisse, concrètement, y marcher.»11

11 Si le système géométral, bidimensionnel, se trouve si bien adapté à la conception et à l'appréhension d'objets architecturaux, donc nécessairement tridimensionnels, c'est qu'il ne s'agit pas ici de maitriser un espace cartésien continu et indifférencié mais l'espace terrestre qui est hétérogène et qualifié : «Géométriquement, les trois coordonnées du système cartésien de l'espace sont d'importances et de propriétés égales. Notre espace terrestre, toutefois, est entièrement soumis à l'attraction gravitationnelle qui particularise la verticale comme la direction de référence. Toute autre orientation spatiale est perçue par rapport à la verticale.» ${ }^{12}$.

12 Maurice Merleau-Ponty a évoqué lui aussi le rôle primordial dans le « vis-à-vis du sujet et du monde» de la verticale et de l'horizontale qui «se définissent en dernière analyse par 
la meilleure prise de notre corps sur le monde. Largeur et hauteur comme relations entre des objets sont dérivées et dans leur sens originaire elles sont des dimensions existentielles.»13.

13 La représentation en géométral est précisément fondée sur cette distinction entre vertical et horizontal et c'est à ce titre qu'elle constitue un moyen d'opérer sur ce que Merleau-Ponty ou Christian Norberg-Schultz ${ }^{14}$ nomment «l'espace existentiel» des hommes.

\subsection{La contrainte de segmentation des vues : focalisations dans la figuration.}

En fait, chaque élément du géométral permet au concepteur de centrer délibérément son attention sur un pôle intentionnel donné relatif au projet. L'étude de la disposition d'un édifice, par exemple, - c'est à dire l'étude des relations, des proportions, de l'assemblage de ses différents constituants - sera déterminée par un travail sur son dessin en plan ; et l'on ne voit pas très bien quel autre type de représentation pourrait posséder la même efficacité dans ce domaine. Car, comme le note Arnheim, «bien que la représentation complète d'un bâtiment exige une intégration tridimensionnelle, sa réduction au plan représente plus qu'une simple commodité technique. Les avantages sont considérables. Par ces deux dimensions extraites de l'ensemble, toutes les proportions et toutes les relations peuvent être rendues correctement. Rien n'est caché, tout est accessible à l'oeil.»1

15 Il est aisé d'extrapoler ce que dit Arnheim à propos de la composition à la plupart des autres domaines de préoccupation sur le projet, par exemple: fonction, distribution, orientation etc. pourront être étudiées principalement à partir d'un travail sur le plan ; volumétrie intérieure, éclairement, structure etc. correspondront à un travail dans lequel les coupes joueront un grand rôle ; volumétrie extérieure, plastique, problèmes perceptifs etc. pourront être abordés sur les élévations. C'est par exemple ce qu'exprime, d'une façon certes un peu caricaturale, Charles Blanc lorsqu'il affirme: "A ces trois termes: convenance, solidité, beauté, correspondent trois opérations de l'architecture ; le plan, la coupe, l'élévation.» ${ }^{16}$.

Nous pouvons préciser encore le mode d'utilisation du géométral dans la conception ou dans l'analyse des édifices en disant que si chacun de ses éléments (plan, coupe, élévation) peut permettre de cerner une catégorie de problèmes, tous ne possèdent pas la même valeur opératoire dans ce domaine. La représentation en plan, comme l'affirme F.L. Wright, joue un rôle prépondérant et semble toujours conserver un caractère dominant dans le travail de figuration architecturale par rapport aux représentations verticales (coupes ou élévations).

Rudolf Arnheim fournit une explication convaincante de ce phénomène de prééminence du plan en remarquant qu'une "projection horizontale possède une complétude dont aucune section verticale ne peut se prévaloir. Car le plan couvre l'étendue totale de l'espace dans lequel l'homme se déplace. L'espace représenté sur un plan est intégral dans la mesure où la troisième dimension n'est pas perçue comme manquant au tableau.[...] Une élévation ne possède jamais cette complétude. Alors que le plan, comme une carte, peut être vu sous tous ses angles, l'élévation établit intrinsèquement une distinction 
entre verticalité, horizontalité et obliquité ; seule la dimension verticale y est totalement significative.» ${ }^{17}$.

18 Ce sont donc bien à la fois les propriétés relatives à son caractère bidimensionnel et fragmentaire qui déterminent, pour une grande part, la valeur opératoire du système plan/coupe/élévation pour la compréhension du projet. Car, encore une fois, tout se passe ici comme si «l'objectivité» du projet ne résidait pas principalement dans son appréhension tridimensionnelle : «Il est [...] admirable, remarque Arnheim, que l'esprit humain soit capable de construire une image de forme objective à partir de vues isolées. Bien des gens peuvent se représenter avec une certaine précision un cube complet bien qu'on ne puisse jamais percevoir plus de trois côtés d'un cube à la fois. Une telle image mentale est nécessairement composée à partir de visions fragmentaires dont aucune n'est contenue dans la forme »objective« d'un cube.[...] Dès lors une oeuvre architecturale est un objet que personne n'a jamais vu et ne verra jamais dans sa totalité. C'est une image mentale, synthétisée avec plus ou moins de succès, à partir de visions partielles. La facilité ou la difficulté que l'on a à construire cette image dépend des formes utilisées par l'architecte. $)^{18}$.

Les outils de description d'un édifice ne doivent donc pas simplement permettre à l'architecte de le représenter mais plus que tout autre chose de se le représenter "mentalement», et c'est pourquoi le géométral possède un efficacité particulière pour la compréhension du projet. Il remplit une fonction heuristique majeure dans le travail de conception architecturale par cette individualisation des figurations qu'il autorise.

Il serait néanmoins inexact de prétendre que le géométral permet de négliger la question tridimensionnelle dans la représentation du projet. Il est indéniable, par exemple, que le recours à ce système de figuration suppose une certaine maîtrise chez l'architecte de l'articulation, de la correspondance spatiale entre plan, coupe et élévation. Ce que nous avons souligné jusqu'ici c'est que cette maîtrise ne constitue pas un facteur plus déterminant qu'un autre pour l'efficacité d'utilisation du géométral et que par ailleurs le travail sur le projet par focalisations intentionnelles qu'autorise le géométral est une clef de cette efficacité.

21 On peut noter en effet que chacune des projections du système géométral prise isolément (plan ou coupe etc.) recèle une certaine autonomie informationnelle et rend compte par elle même de certaines caractéristiques spatiales de l'objet décrit. Ainsi, par exemple, la dimension verticale n'est jamais totalement absente de la représentation par projection géométrale en plan. On distingue en effet par leur codification particulière (pochage, épaisseur de trait...) les ouvrages physiques sectionnés de l'ensemble des objets situés en arrière du plan de section. Le plan réalise donc l'articulation de ces deux catégories d'informations (section et arrière plan) en vue de préciser certains aspects de l'organisation verticale de l'édifice.

Cette prise en compte de propriétés spatiales d'un bâtiment par son plan de géométral offre l'illustration d'une caractéristique plus générale et remarquable de ce système de représentation. Celle-ci tient dans le fait que la focalisation que le géométral permet sur chaque domaine de préoccupation relatif au projet ne consiste pas exactement en une stricte décomposition analytique par isolements successifs des éléments signifiants pour ce domaine. Ces focalisations sont loin d'être " étanches»; elles s'opèrent par une mise en relief d'un niveau de signification du projet compte tenu des autres, ce qui correspond bien au sens de ce mot tel que le définit la sémiologie : «la focalisation consiste [non à 
isoler mais] à mettre en relief (en focus) un élément d'un énoncé par des moyens propres au code employé. ${ }^{19}$.

Le géométral correspond ainsi à une représentation du projet par fragmentation apparente dans laquelle, pourtant, chaque pièce du système de représentation permet d'isoler certains pôles de préoccupation (volume, structure, fonction, composition...) sans les déconnecter simultanément des autres qui remplissent alors un rôle de contextualisation de la réflexion sélective propre à la conception ou à l'interprétation du projet. $^{20}$

Nous résumerons cela en disant que le géométral correspond à un système d'information traditionnel pour l'architecture qui est caractérisé par des propriétés que l'on pourrait qualifier d'intercontextuelles (possibilités de focalisations/contextualisations); ces propriétés semblent jouer un rôle non négligeable dans l'adaptabilité de l'outil géométral aux tâches de la conception et de l'analyse architecturales.

\section{La conception par dissensus}

Plusieurs expérimentations que nous avons réalisées dans le cadre d'enseignements de différents formats montrent la valeur opératoire du principe de dissensus ${ }^{21}$ pour la didactique de la conception et de la représentation architecturale. La représentation dissensuelle fragmente ses outils et discrétise ses procédures descriptives. À l'instar par exemple du géométral de l'architecte ${ }^{22}$, elle s'appuie sur un principe de séparation critique des supports de représentation.

Dans la représentation de dissensus, ce sont les conflits, les collisions et les dissociations qui opèrent, on y propose de circuler de représentations en représentations, comme de mondes en mondes, sans liens déterminés, sans ressemblances prévues. Plus ces mondes sont étrangers et plus la collision promet d'être productive. C'est dans ce sens que Jacques Rancière écrit : «Ce que j'entends par dissensus n'est pas le conflit des idées ou des sentiments. C'est le conflit de plusieurs régimes de sensorialité $»^{23}$. Organiser les conflits, favoriser les contradictions. Pour le concepteur, cela signifie privilégier l'usage de dispositifs hétérogènes- physiques, numériques, sensibles, logiques, narratifs, etc.- qui activent des régimes de représentation contradictoires. Multiplicité et dissensus visent alors à enrichir la saisie du réel dans toute sa complexité

Par ailleurs, si nous admettons l'idée que la conception fonctionne sur le modèle d'une " conversation réflexive avec la situation », il faut alors que ce processus critique s'appuie sur des points de divergences et des paradoxes actifs capables d'élargir la perception de la réalité chez le concepteur.

Le principe dissensuel en conception repose donc sur la disjonction des descriptions de la réalité. Il se manifeste d'ailleurs souvent par l'usage de l'image paradoxale, ou de la métaphore. En matière d'enseignement de la conception, c'est aussi la compétence d'un professionnel expérimenté que de savoir produire les images critiques susceptibles de restructurer temporairement la situation de conception par des ruptures signifiantes pour l'étudiant. Comme l'écrit Seymour Papert lui-même, la capacité de redescription de l'action est le propre du bon formateur qui «sait capturer la complexité d'une action dans des métaphores (par exemple 'affrontez la pente!') qui aident à transmettre la sensation d'une performance à réaliser. ${ }^{24}$ 
Les outils de représentation en conception architecturale jouent ce rôle re-descriptif et re-structurant des situations d'action lorsqu'ils stimulent les interprétations chez l'architecte. En créant des possibilités de décentrement du concepteur dans son travail ce sont ces outils en effet qui offrent l'un des supports de "l'enquête de métier» par laquelle le professionnel examine, répertorie, expérimente et explore de façon exhaustive la situation de conception. A partir de là, assumer le principe de dissensus en matière de représentation architecturale signifie d'abord garantir l'hétérogénéité et la divergence des représentations manipulables.

Plus généralement une telle prise en compte des pratiques interprétatives dans le processus de conception installe des notions comme l'incertitude ou la subjectivité du sujet concepteur au centre des réflexions.

1 Le principe de dissensus rend aussi problématique la question des assistances techniques à la représentation dans la mesure où celle-ci devrait plutôt consister à augmenter les divergences descriptives au lieu de les résoudre ou bien de les cacher. Dans ce contexte, le rôle du concepteur évolue, on attendra moins de lui une formalisation de la situation qu'une interprétation des différents systèmes d'informations et de ressources présents, ou encore une mise en relation signifiante de données hétérogènes et contradictoires. De même, son expertise d'architecte correspondra moins ici à celle d'une maîtrise technique directe des processus d'édification qu'à celle d'une connaissance " méta-technique » des situations hétérogènes (information, usages, ressources, histoires...).

Les écrits de l'architecte Yona Friedman sur l'auto planification, ont rendu compte avec précision des conséquences sociales de ce type d'intervention de conception dans laquelle l'architecte n'est plus concepteur de détail mais devient consultant, organisateur, personne ressource fournissant des connaissances en écologie, en construction, en organisation, en histoire ${ }^{25}$. Dans cette hypothèse, on voit que l'individu concepteur est pris dans une constellation de conception plus vaste que lui-même et dans laquelle interviennent toutes les ressources de son environnement. C'est cette distribution cognitive que le chercheur David N. Perkins a nommé l'individu-plus ("person-plus») : «L'environnement - à savoir les ressources sociales et physiques situées dans l'environnement immédiat en dehors de l'individu - fait partie de la cognition, non pas seulement comme source ou récepteur d'information, mais également comme véhicule de la pensée. $»^{26}$

Sans doute toutes ces voies de recherche visent-elles à considérer des démarches de conception fragmentées et distribuées. Aussi proposent-elles implicitement d'intégrer par exemple une certaine dose «d'expertise démocratique» collective dans la conception. Comme nous venons de le souligner, la recherche de décentrement du concepteur en cours de conception est sous tendue par la notion de représentation dissensuelle. Une telle pratique risque de modifier la représentation que ce dernier a de son rôle professionnel et de le placer face à des tensions contradictoires. Pour la conception en architecture, ces contradictions concernent notamment les rapports entre formalisation des fonctions et improvisation des pratiques, entre protocoles et transgressions, entre assistance technique et émancipation pratique, entre architecte concepteur et architecte méta-concepteur et plus généralement entre ouverture et fermeture disciplinaire de l'enseignement de la conception architecturale. 
n'est-ce pas précisément par l'étude de ces contradictions qu'il devient possible de proposer des outils autres pour une conception de l'architecture contemporaine qui assume mieux le modèle d'un concepteur subjectif et agissant?

\section{BIBLIOGRAPHIE}

Bibliographie.

Arendt, H. Condition de l'homme moderne. Calmann-Lévy, 1958, ré-éd. Pocket Agora, 1994, Paris.

Arnheim, R. Dynamique de la forme architecturale. Pierre Mardaga éditeur, 1986, Bruxelles.

Benjamin, W. « L'oeuvre d'art à l'époque de sa reproduction mécanisée» in Écrits français, 1936, Éditions Gallimard, 1991, Paris.

Bidault, C. Modes d'influence des techniques de représentation sur le processus de conception architecturale. diplôme de troisième cycle, École Régionale d'Architecture de Nancy, 1975, Nancy.

Boudon, P. et Pousin, F. Figures de la conception architecturale. Éditions Dunod, 1988, Paris.

Castex, J. Frank Lloyd Wright, le Printemps de la Prairie House. Pierre Mardaga éditeur, 1985, Bruxelles.

Deforge, Y. Le Graphisme Technique son histoire et son enseignement. Éditions du Champ Vallon (collection milieux), 1981, Mâcon.

Estevez D. « Le concepteur émancipé. Dissensus et conception en architecture » in 01Design 8 Echelles, Espaces, Temps, actes du colloque, Académie Royale des Beaux-Arts de Bruxelles, 9-11 mai 2012

Estevez D. Dessin d'architecture et infographie, nouvelles pratiques graphiques, CNRS Editons, 2001, Paris, p. 95

Estevez, D. Les fonctions traditionnelles de la figuration architecturale à l'époque des outils informatiques. Rapport de recherche Li2a, École d'Architecture de Toulouse, 1999, Toulouse.

Estevez, D. Étude d'un modèle d'interprétation du dessin géométral en phase de conception du projet d'architecture. Diplôme d'ingénieur, Conservatoire National des Arts et Métiers, 1995, Toulouse.

Estevez, D. « Compte rendu d'une étude pour un outil logiciel d'analyse du dessin géométral en architecture» in Un bilan comparatif, apport de la programmation orientée objet et des systèmes à bases de connaissances en CAO en architecture Workshop, conférence Europ'IA 96, 1996, Lyon.

Freidman Y. L'architecture de survie, une philosophie de la pauvreté, Eds L'Eclat, 2003, Paris

Holtz-Bonneau, F. Lettre, image, ordinateur. Hermès/INA, 1987, Paris.

Joly, M. L'image et les signes. Approche sémiologique de l'image fixe. Éditions Nathan, collection Image, 1994, Paris.

Lauret, G. Informatique graphique dans le bâtiment et l'architecture. Masson, 1990, Paris.

Léglise, M. « Art Under Constraint, Preserving the creative dimension in computer aided architectural design» in Languages of Design, 3, Elsevier, 1995, Amsterdam 
Martin, J. C. L'image virtuelle. Essai sur la construction du monde. Éditions Kimé, 1996, Paris.

Merleau-Ponty, M. Phénoménologie de la perception. Éditions Gallimard, 1945, réédition Tel, 1992, Paris.

Morin, E. La Méthode 1. La nature de la nature. Éditions du Seuil, 1977, Paris.

Norberg-Schulz, C. Genius Loci. Éditions Dessert \& Mardaga, 1972, Bruxelles.

Quintrand, P., Autrant, J., Florenzano, M., Frégier, M. et Zoller, J. La CAO en architecture. Éditions Hermès, 1985, Paris.

Ranciere J. Les paradoxes de l'art politique in « Le spectateur émancipé », La Fabrique éditions, Paris, 2008

Rabardel, P. « Représentations dans les Situations d'Activités Instrumentées» in Représentations pour l'action, Octares Éditions, 1993, Toulouse.

Séris, J. P. La technique. Presses Universitaires de France, 1994, Paris.

Schön D.A. « The reflexive practitionner. How professionals think in action. », Basic Books Inc. 1983, New York

Simon, H. A. Sciences des systèmes. Sciences de l'artificiel. Bordas, collection Dunod-Afcet Systèmes, 1991, Paris.

Weisbuch, G. Dynamique des systèmes complexes. InterEditions/Éditions du CNRS, collection Savoirs Actuel, 1989., Paris.

Zeitoun, J. Trames planes. Introduction à une étude architecturale des trames, Éditions Bordas, 1977, Paris.

\section{NOTES}

1. [Rabardel, P. 1993], p.107

2. [Deforge Y. 1981], p.202

3. [Bidault C. 1975], p.4

4. [Quintrand P. et al. 1985], p.85

5. Nous avons développé cette idée lors d'une étude précédente [Estévez D. 1995], pp.8-13

6. [Castex J. 1985], p.90

7. Frank Lloyd Wright, Première version de "In the cause of Architecture", 1908, cité par [Castex J. 1985]

8. [Arnheim R. 1986]

9. [Arnheim R. 1986], p.61

10. [Arnheim R. 1986], p.62

11. [Benjamin W. 1991], p.190

12. [Arnheim R. 1986], p.43

13. [Merleau-Ponty M. 1992], p.309 et cette phrase intéressante sur le même sujet : "Renverser un objet c'est lui ôter sa signification.", p.292

14. [Norberg-Schultz, C. 1972]

15. [Arnheim R. 1986], p.64

16. Charles Blanc "Grammaire des arts du dessin" cité par [Bidault C. 1975], p.50

17. [Arnheim R. 1986], p.70

18. [Arnheim R. 1986], p.116

19. [Joly M. 1994], p.83 
20. Sur les notions de contextualisation de l'interprétation du dessin d'architecture auxquelles j'ai consacré plusieurs travaux, on pourra se reporter à leur résumé dans [Estévez D. 1995].

21. [Estevez D. 2012]

22. [Estevez D. 2001] p. 95

23. [Ranciere J.] 2008, p.62, p.66.

24. Papert Seymour cité par [Schön DA. 1983] p.279

25. FREIDMAN Y. (2003), «L'architecture de survie, une philosophie de la pauvreté », Eds L'Eclat, Paris.

26. PERKINS David N. (1995) «L'individu-plus. Une vision distribuée de la pensée et de l'apprentissage. » In: Revue française de pédagogie. Volume 111, 1995. pp. 57-71.

http://www.persee.fr/web/revues/home/prescript/article/

rfp_0556-7807_1995_num_111_1_1232

\section{RÉSUMÉS}

Le travail que nous présentons ici concerne l'étude des modalités contemporaines de figuration graphique en architecture. La démarche qui y est adoptée assume une analyse critique des outils traditionnels de figuration. La notion de fragmentation dans le travail graphique de constitue le support de cette réflexion critique. Elle peut permettre de dégager un principe de représentation dissensuelle propre au travail de conception en architecture.

\section{AUTEUR}

\section{DANIEL ESTEVEZ}

Groupe Art Architecture Conception du laboratoire LRA

École Nationale Supérieure d'Architecture de Toulouse

daniel.estevez@toulouse.archi.fr 